\title{
Caracterización de decomisos de vísceras rojas en un frigorífico de exportación en Santander - Colombia
}

\author{
Characterising red viscera confiscation in an export slaughterhouse \\ in Santander, Colombia
}

\section{Caracterização de confiscos de vísceras vermelhas num frigorífico de exportação em Santander - Colômbia}

\author{
Felipe Ramírez-Londoño ${ }^{1}$, Arturo Cárdenas-Pinto ${ }^{2 *}$, Víctor Arcila-Quiceno ${ }^{3 *}$, Ruby Cristancho ${ }^{4}$, \\ Jeiczon E. Jaimes-Dueñez ${ }^{*}$ \\ 1 MVZ, Frigorífico Río Frío (FRF), Vía corredor Río Frío Calle 210 N 9 - 631, Floridablanca, Santander, Colombia \\ 2 MVZ,Esp \\ 3 MVZ, Esp, MSc \\ 4 Microbiol de Alimen, COLBEEF \\ 5 MVZ, MSc, PhD \\ * Grupo de Investigación en Ciencias Animales (GRICA), Facultad de Medicina Veterinaria y Zootecnia, \\ Universidad Cooperativa de Colombia (UCC), Calle 30 No. 33 - 51, Bucaramanga, Santander, Colombia \\ Email: jeiczon.jaimes@campusucc.edu.co
}

Recibido: 27 de enero de 2020

Aceptado: 27 de abril de 2020

\section{Resumen}

A nivel mundial el decomiso de vísceras rojas es una de las principales causas de pérdidas económicas en la comercialización de productos cárnicos. El objetivo del presente estudio fue determinar las principales causas y variables epidemiológicas asociadas al decomiso de vísceras rojas en el FRIGORíFICO RÍO FRÍO (FRF), del municipio de Floridablanca, Santander y estimar el valor de las pérdidas económicas asociadas a esta actividad. Entre 9 de noviembre al 9 de diciembre de 2018, se realizó un estudio transversal en el total de animales sacrificados en el FRF, a los cuales se les recolectó información epidemiológica posterior a la inspección sanitaria realizada por funcionarios del INVIMA. Las principales causas y variables epidemiológicas asociadas al decomiso de vísceras rojas se estimaron a partir de análisis de frecuencias, mientras el valor de las pérdidas económicas, a través de un análisis económico en el mercado nacional. Se sacrificaron un total de 8671 bovinos, y 226 búfalos, en los cuales se realizó el decomiso de vísceras rojas en el 15,8\% y 26,5\% de los animales, respectivamente. En los bovinos los principales órganos decomisados fueron pulmón, hígado y bazo con porcentajes del 87,4\%, 11,1\% y $0,6 \%$, respectivamente. Mientras que, en los búfalos, fueron el pulmón, hígado y riñón con porcentajes del 88,3\%, 8,3\% 
y $1,7 \%$, respectivamente. Las principales causas de decomiso de pulmón en ambas especies fueron la broncoaspiración $(86,6 \%)$ y enfisema $(9,4 \%)$, mientras para el hígado fueron los abscesos (40,5\%) y la distomatosis hepática (28,8\%) en los bovinos, hígado graso $(80,0 \%)$ y cirrosis $(20,0 \%)$ en los búfalos. Para el caso de los bovinos, hubo diferencias significativas en la proporción de animales con distomatosis hepática entre los diferentes municipios de Santander $\left(\chi^{2}=50.03\right.$, P < 0.05). Las mayores pérdidas económicas durante el periodo de estudio se deben al decomiso de pulmón e hígado con valores de 4886 y 2639 USD. En conclusión, el pulmón fue el órgano decomisado con mayor frecuencia en ambas especies, principalmente asociado a la broncoaspiración. Futuras estrategias de intervención, con el fin de reducir la broncoaspiración en los animales que ingresan a la planta de beneficio, e interrumpir el ciclo de vida de Fasciola hepatica en algunos municipios de Santander, son necesarios para disminuir el nivel de pérdidas económicas a lo largo de la cadena productiva.

Palabras clave: bovinos; búfalos; Colombia; hígado; lesiones; pulmón; sacrificio

\begin{abstract}
Red viscera confiscation represents a significant cause of economic loss for the meat products' trade. This study was aimed at determining the causes and epidemiological variables associated with red viscera confiscation in a slaughterhouse (FRIGORÍFICO RÍO FRÍO (FRF)) located in Floridablanca, in Colombia's Santander department, and estimating the value of the economic losses associated with such confiscation. A cross-sectional study was carried out between November and December 2018 based on epidemiological information regarding all animals slaughtered at the FRF recorded after routine health inspection by Colombian Food and Drug Surveillance Institute (INVIMA) officials. Frequency analysis was used for estimating the main causes of and the epidemiological variables associated with red viscera confiscation whilst economic losses were estimated by national market analysis. It was found that red viscera were confiscated in $15.8 \%$ of 8,671 sacrificed cattle compared to $26.5 \%$ for 226 buffaloes. The lungs $(87.4 \%)$, liver $(11.1 \%)$ and spleen $(0.6 \%)$ were the main organs seized for cattle and lungs $(88.3 \%)$, liver $(8.3 \%)$ and kidneys $(1.7 \%)$ for buffaloes. Bronchoaspiration $(86.6 \%)$ and emphysema $(9.4 \%)$ were the main causes of confiscation regarding the lungs in both species while for the liver these were abscesses (40.5\%) and hepatic dystomatosis $(28.8 \%)$ in cattle and fatty liver $(80.0 \%)$ and cirrhosis $(20.0 \%)$ in buffaloes. There were significant differences regarding the percentage of cattle having hepatic dystomatosis amongst municipalities in the Santander department $\left(\chi^{2}=50.03\right.$, $\mathrm{p}<0.05)$. Lung $(4,886$ USD) and liver confiscation $(2,639$ USD) led to the greatest economic losses; the lungs were the main organ confiscated in both species, mainly being associated with bronchoaspiration. Future intervention strategies are needed to reduce the livestock industry's financial losses, aimed at reducing bronchoaspiration in animals in the slaughterhouse and interrupting Fasciola hepatica's life-cycle in some municipalities in the Santander department.
\end{abstract}

Keywords: cattle; buffalo; Colombia; liver; lesion; lung; slaughter.

\title{
Resumo
}

Globalmente, os confiscos de órgãos e vísceras vermelhas é uma das principais causas de perdas econômicas na comercialização de produtos à base de carne. O objetivo deste estudo foi determinar as principais causas e variáveis epidemiológicas associadas aos confiscos de vísceras vermelhas num frigorífico (FRIGORÍfICO RÍO FRÍO (FRF)), do município de Floridablanca, Santander, e estimar o valor das perdas econômicas associadas a esta atividade. Entre 9 de novembro e 9 de dezembro do 2018, foi realizado um estudo transversal sobre o total de animais abatidos no FRF, nos quais a informação epidemiológica era diligenciada depois da inspeção sanitária realizada pelos funcionários do INVIMA. As principais causas e variáveis epidemiológicas associadas ao confisco de vísceras vermelhas foram estimadas a partir da análise de freqüência, e o valor de perdas econômicas, através de uma análise no mercado nacional. Foram abatidos 8.671 bovinos e 226 búfalos, nos quais 15,8\% e 26,5\% tiveram confiscos de vísceras vermelhas, respectivamente. Em bovinos, as principais vísceras confiscadas, foram pulmões, fígado e baço com percentagens de $87,4 \%, 11,1 \%$ e 0,6\%, respectivamente. Nos búfalos, foram pulmões, fígado e rim com porcentagens de 88,3\%, 8,3\% e 1,7\%, respectivamente. Para as duas espécies, broncoaspiração $(86,6 \%)$ e enfisema $(9,4 \%)$ foram as principais causas de confiscos dos pulmões. Para o fígado, foram os abcessos $(40,5 \%)$ e fasciolose $(28,8 \%)$ em bois, esteatose hepática $(80,0 \%)$ e cirrose $(20,0 \%)$ em búfalos. No caso dos bois, houve diferenças significativas na proporção de animais com fasciolose nos diferentes municípios do Santander $\left(\chi^{2}\right.$ $=50,03, \mathrm{P}<0,05)$. As maiores perdas econômicas durante o período de estudo correspondem ao confisco de pulmão e fígado com valores de 4886 e 2639 USD. Em conclusão, o pulmão foi a principal víscera confiscada em ambas espécies, principalmente associados a broncoaspiração. Estratégias futuras de intervenção, com o objetivo de reduzir a broncoaspiração, e interromper o ciclo de vida da Fasciola hepatica em alguns municípios do Santander são necessários para reduzir as perdas econômicas na cadeia de produção.

Palavras-chave: gado; búfalo; Colômbia; fígado; lesões; pulmão; abate

\section{Introducción}

Colombia es el tercer país más ganadero en Suramérica, seguido de Brasil y Argentina (Contexto Ganadero, 2015), con un total de 27234027 cabezas de bovinos y 308580 cabezas de búfalos reportadas para el año 2019 (ICA, 2017). En América el decomiso de vísceras rojas en las plantas de beneficio es una de las causas principales que explican pérdidas económicas asocia- 
das a la comercialización de productos cárnicos. En Brasil y Costa Rica se estima que las pérdidas económicas oscilan alrededor de los 10000 USD mensuales, por planta de beneficio (Burin et al., 2013; Rojas y Cartín, 2016), mientras que en Colombia se han estimado pérdidas de hasta 71000 USD en departamentos del suroeste del país (Cedeño et al., 2012).

En Colombia existen cerca de 200 plantas de beneficio animal (INVIMA, 2018). Cinco de ellas, exportan principalmente hacia Chile, Rusia y Curazao: frigorífico FRIGOCOLANTA ubicado en el departamento de Antioqua, MINERVA FOODS y FRIGOSINÚ ubicados en el departamento de Córdoba, VIJAGUAL y RÍO FRÍO ubicados en el departamento de Santander. Un reciente estudio realizado en frigoríficos del departamento de Córdoba concluyó que la principal causa de decomiso de pulmones en bovinos se debe a fallas en el proceso de insensibilización y sacrificio de los animales, así como posibles procesos infecciosos (Oviedo et al., 2016); no obstante, los factores epidemiológicos asociados al decomiso de otros órganos tanto en bovinos y búfalos, al igual que su impacto económico en la producción, permanecen sin ser conocidos en este país.

Actualmente, el FRIGORÍFICO RÍO FRÍO S.A.S (FRF), reporta un alto número de decomisos de vísceras rojas, con sus consecuentes impactos económicos. Mensualmente la cantidad de decomisos alcanza hasta el 20\% de los animales sacrificados, con pérdidas anuales hasta de 147468 USD, que afectan significativamente la ganancia de los productores y obstaculizan la posibilidad de exportación y comercialización en países extranjeros (Ramírez, 2019). Entre las principales causas de decomiso, se encuentran las enfermedades subdiagnosticadas, residuos de medicamentos y procesos fisiopatológicos asociados al tiempo y estrés del transporte, al igual que los tiempos de espera en la planta de beneficio. A pesar de esta importante casuística, el FRF no cuenta con una caracterización detallada de los decomisos de vísceras rojas, por lo que el desarrollo de estrategias de prevención que reduzcan el impacto económico de esta problemática continúa siendo poco explorado. En este sentido, los objetivos del presente estudio consistieron en determinar las principales causas y variables epidemiológicas asociadas al decomiso de vísceras rojas en el FRF, y estimar el nivel de pérdidas económicas asociadas a estos eventos.

\section{Metodología}

\section{Descripción del área de estudio}

El presente estudio se llevó a cabo en el FRF, el cual es una empresa legalmente conformada desde el año
2013, ubicada en el municipio de Floridablanca, Santander (FRF, 2020). Dicha planta, cuenta con la capacidad de alojar un total de 2059 animales al día, en 103 corrales de espera, una capacidad de sacrificio de 450 animales en 8 horas, con un beneficio promedio de 8000 animales al mes (Ramírez, 2019).

\section{Tipo de estudio y muestreo}

El presente estudio consistió en un estudio descriptivo de corte transversal, en el cual el grupo muestral se integró con los individuos beneficiados en el FRF, durante el período comprendido entre el 9 de noviembre y el 9 de diciembre de 2018. El grupo muestral se integró con los individuos que presentaron lesiones macroscópicas evidentes y terminaron en decomiso según lo establecido en la resolución 240 de 2013 (Minsalud, 2013).

\section{Proceso de pre faenado, faenado e inspección de vísceras rojas}

Luego de la llegada de los animales al FRF en camiones transportadores, estos fueron descargados por medio de rampas, ubicados en corrales techados durante un periodo de máximo 48 horas, y mantenidos con agua ad libitum durante la espera. Antes de entrar al proceso de faenado los animales fueron sometidos a una inspección ante-mortem realizada por funcionarios del Instituto Nacional de Vigilancia de Medicamentos y Alimentos (INVIMA), quienes otorgaban el aval para el proceso de sacrificio.

La llegada de los animales al área de sacrificio se realizó a través de una manga de movilización por medio de la cual llegaban a un cajón de noqueo hidráulico, en donde ocurría la insensibilización del animal con un aturdidor neumático penetrante (Jarvis ${ }^{\circledR}$ Campiñas, Brasil). Posteriormente los animales eran izados y pasados a un sistema de rieles, en el cual se iniciaba el desangrado del animal, desuello manual de piernas y brazos, y el desuello total de la canal de forma mecanizada. Enseguida ocurría la evisceración (vísceras blancas y rojas), separación de canales e inspección sanitaria post-mortem por parte de los funcionarios del INVIMA, previa liberación de las canales para su posterior pesaje y almacenamiento.

La inspección de vísceras rojas se realizó según lo señalado en la resolución 240 de 2013 a cargo del INVIMA (Minsalud, 2013). Inicialmente se realizaba inspección general del paquete visceral y posteriormente inspección detallada de cada una de ellas (Corazón, pulmón, hígado, bazo y riñones). En resumen, la inspección sanitaria se basó en la verificación del sistema 
de trazabilidad, examen visual, palpación, e incisión de órganos, con el objetivo de asegurar la ausencia de enfermedades o defectos visibles en las vísceras, así como reducir al mínimo la contaminación biológica, química y física, de estas.

\section{Caracterización de la información}

Después de la inspección a cargo del INVIMA, a los animales que presentaron decomiso de vísceras rojas se les elaboró un registro de algunas variables epidemiológicas como el tiempo de transporte ( 1 a 5 horas, 6 a 10 horas, 11 a 15 horas y $>16$ horas), tiempo de espera en corrales ( 6 a 15 horas, 16 a 30 horas, 31 a 45 horas $y>46$ horas), raza (Bos primigenius indicus, Bos primigenius taurus y cruces), sexo (hembra y macho), procedencia (departamento y municipio), edad (años), condición corporal (escala de 1 a 5), órgano decomisado (bazo, corazón, hígado, pulmón, riñón y víscera completa), patología macroscópica y calificación de grado (leve, moderado o grave). Finalmente, la temperatura visceral fue tomada en un tiempo no mayor a 20 minutos luego del sacrificio del animal utilizando un termómetro digital (Hanna ${ }^{\circledR}$, Rhode Island, Estados Unidos).

Los análisis de frecuencias fueron realizados en el programa Excel v. 19.0. La comparación del número de animales decomisados entre especies, y el número de decomisos entre animales de diferentes procedencias fue analizado mediante la prueba de chi-cuadrado $\left(\chi^{2}\right)$ en el programa SPSS v.23.0. Un $P<0.05$, fue considerado significativo. Las gráficas fueron realizadas en el programa Prism v. 8.1.2.

\section{Evaluación económica}

Con el fin de estimar las pérdidas económicas asociadas al decomiso de vísceras rojas, el costo promedio de cada víscera decomisada (CV) fue estimado multiplicando el peso promedio de cada órgano en kilogramos (PV) según Xiaojuan et al., 2016, por el precio oficial promedio (PO) de cada kilogramo de víscera (USD) publicado en los principales almacenes de cadena del país durante el segundo semestre del 2019 (Almacenes Éxito Inversiones S.A.S, Cencosud Colombia S.A.S, y Carulla Vivero Holding INC) (fórmula 1). El valor mensual de las pérdidas económicas asociadas al decomiso de cada víscera en el FRF (PPCV) se estimó a partir del costo promedio de cada víscera decomisada $(\mathrm{CV})$, multiplicado por el total de unidades de dicho órgano decomisados durante el mes en estudio (TVDM) (fórmula 2). Las pérdidas económicas por cada animal con decomi- so independiente del órgano decomisado (PPAD), se estimó a partir del valor total de pérdidas por decomisos (TPPD), dividido por el total de animales decomisados durante el periodo de estudio (TAD) (fórmula 3). En cuanto a la tasa de cambio utilizada en el presente estudio, se estableció que 1 dólar americano (USD) equivale a \$3409 colombianos (Dólar-Colombia, 2018).

$$
\begin{array}{ll}
\text { F1. } & C V=P V \times P O \\
\text { F2. } & \text { PPCV }=\text { CV } \times \text { TVDM } \\
\text { F3. } & \text { PPAD }=\text { TPPD } / \mathrm{TAD}
\end{array}
$$

Formulas. F1, F2 y F3, corresponden a la fórmula 1, formula 2 y formula 3, empleadas para la evaluación económica. Fuente propia.

\section{Resultados}

\section{Descripción general de los decomisos}

Durante el período de estudio se sacrificaron un total de 8671 bovinos, y 226 búfalos, observándose una menor proporción de decomisos de vísceras rojas en los bovinos $(5,8 \%, 1373 / 8671)$, comparado con los búfalos $(26,5 \%, 60 / 226)\left(\chi^{2}=5.45, P<0.05\right)$. En el caso de los bovinos los principales órganos decomisados fueron pulmón, hígado y bazo con porcentajes del $87,4 \%(1200), 11,1 \%$ (153) y $0,6 \%$ (9), respectivamente (figura 1). Para el caso de los búfalos, los órganos más decomisados fueron el pulmón, hígado y riñón con porcentajes del $88,3 \%$ (53), $8,3 \%$ (5) y $1,7 \%$ (1), respectivamente.

\section{Descripción general de pulmones decomisados}

A los bovinos, se les decomisaron 1200 pulmones, siendo la principal causa de decomiso la broncoaspiración $(77,3 \%)$, seguido por el enfisema $(14,8 \%)$, congestión $(4,7 \%)$, neumonía $(2,1 \%)$ y abscesos $(0,5 \%)$ (figura 2A). Mientras que, en los búfalos, se decomisaron 53 pulmones, siendo la principal causa de decomi-

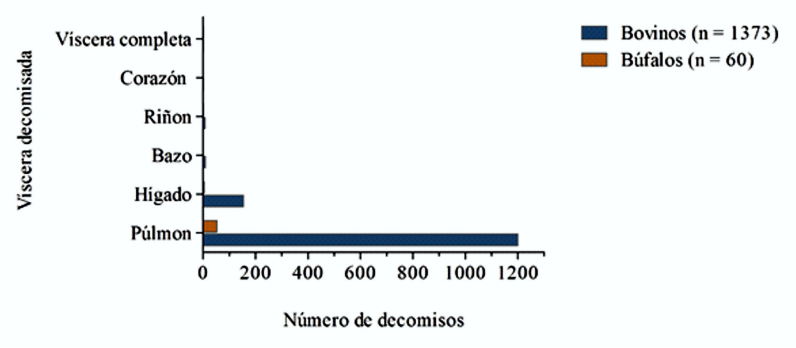

Figura 1. Cantidad de órganos decomisados en bovinos y búfalos durante el estudio realizado en el FRF. Fuente propia. 
A.

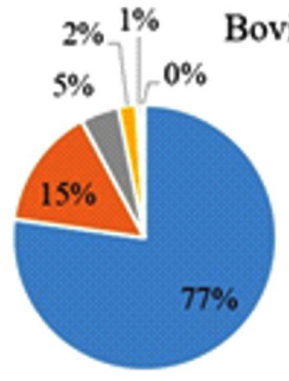

B.

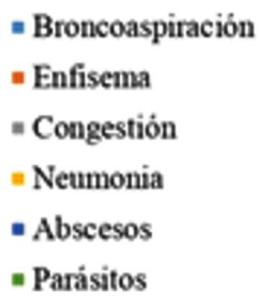

Búfalos

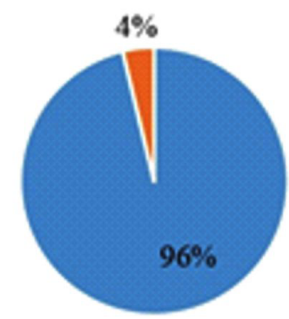

- Broncoaspiración

- Enfisema

Figura 2. Causas de decomisos en pulmones bovinos (A) y bufalinos (B) durante el estudio realizado en el FRF. Fuente propia.

so la broncoaspiración $(96,0 \%)$, y el enfisema $(4,0 \%)$ (figura 2B).

En cuanto a los bovinos que presentaron decomiso de pulmones, las categorías más frecuentes dentro de las variables epidemiológicas analizadas fueron el tiempo de transporte de 6 a 10 horas $(51,3 \%)$, tiempo en corrales de más de 46 horas $(79 \%)$, animales Bos primigenius indicus $(86,3 \%)$, machos $(68,8 \%)$, procedentes del departamento de Santander $(63,5 \%)$, en un rango de edad de 3 a 5 años $(81,2 \%)$, y una condición corporal de 4 sobre $5(56,2 \%)$. Con respecto a la temperatura visceral, se observó que el 1,6\% de los animales con decomiso de esta viscera presentaron una temperatura superior a $40^{\circ} \mathrm{C}$. De otro lado, el grado de lesión más frecuente en los pulmones bovinos decomisados fue de tipo moderado (51,8\%) (tabla 1). Para el caso de los búfalos, las categorías encontradas con mayor frecuencia fueron el tiempo de transporte de 1 a 5 horas $(58,5 \%)$, tiempo en corrales de más de 46 horas $(86,8 \%)$, machos $(92,5 \%)$, procedentes del departamento de Santander $(69,8 \%)$, en un rango de edad de 3 a 5 años $(69,8 \%)$ y una condición corporal de 3 sobre $5(66 \%)$. Con respecto a la temperatura de la canal, no se encontraron animales con temperaturas mayores a $40^{\circ} \mathrm{C}$. El grado de lesión más frecuente en

Tabla 1. Variables epidemiológicas analizadas y categorías encontradas con mayor frecuencia en animales con pulmones decomisados durante el periodo de estudio. Fuente propia.

\begin{tabular}{|c|c|c|c|c|}
\hline \multirow{3}{*}{ Variable } & \multicolumn{4}{|c|}{ Categorías encontradas con mayor frecuencia } \\
\hline & \multicolumn{2}{|c|}{ Bovino } & \multicolumn{2}{|c|}{ Búfalo } \\
\hline & Categoría & $\%$ & Categoría & $\%$ \\
\hline Tiempo transporte & 6 a 10 horas & 51,3 & 1 a 5 horas & 58,5 \\
\hline Tiempo corrales & Más de 46 horas & 79 & Más de 46 horas & 86,8 \\
\hline Raza & Bos indicus & 86,3 & - & - \\
\hline Sexo & Macho & 68,8 & Macho & 92,5 \\
\hline Procedencia & Santander & 63,5 & Santander & 69,8 \\
\hline Edad & 3 a 5 años & 81,2 & 3 a 5 años & 69,8 \\
\hline Temperatura & Mayor de $40^{\circ} \mathrm{C}$ & 1,6 & Mayor de $40^{\circ} \mathrm{C}$ & 0 \\
\hline Condición corporal & $4 / 5$ & 56,2 & $3 / 5$ & 66,0 \\
\hline Patología & Broncoaspiración & 77,3 & Broncoaspiración & 96,2 \\
\hline Grado lesión & Moderado & 51,8 & Grave & 73,6 \\
\hline
\end{tabular}


los pulmones de búfalos decomisados fue de tipo grave $(73,6 \%)$ (tabla 1$)$.

\section{Descripción general de hígados decomisados}

Tanto en bovinos y búfalos, el hígado fue el segundo órgano más decomisado, con un total de 153 y 5 decomisos, respectivamente. Las patologías más frecuentes en los hígados de bovinos fueron los abscesos (40,5\%; 62/153), seguido por distomatosis hepática $(28,8 \% ; 44 / 153)$ y telangiectasia $(17,0 \% ; 26 / 153)$ (figura 3A). Para el caso de los búfalos, las principales causas de decomiso fueron hígado graso $(80,0 \% ; 4 / 5)$ y cirrosis $(20,0 \% ; 1 / 5)$ (figura 3B).

En cuanto a los bovinos que presentaron decomiso de hígado, las categorías más frecuentes dentro de las variables epidemiológicas analizadas fueron el tiempo de transporte de 6 a 10 horas (52,9\%), tiempo en corrales de más de 46 horas (51,0\%), animales Bos primigenius indicus $(64,1 \%)$, machos $(53,6 \%)$, procedentes del departamento de Santander (76,5\%), en un rango de edad de 3 a 5 años (76,5\%) y condición corporal de 3 sobre $5(80,4 \%)$. Respecto a la temperatura de la canal se observó que el $44 \%$ de los animales con decomiso de esta víscera presentó temperaturas mayores a $40^{\circ} \mathrm{C}$. De otro lado el grado de lesión más frecuente en los hígados de bovinos decomisados fue de tipo moderado $(79,1 \%)$. Para el caso de los búfalos las categorías encontradas con mayor frecuencia fueron el tiempo de transporte de 1 a 6 horas $(60,0 \%)$, tiempo en corrales de 16 a 30 horas $(60,0 \%)$, hembras $(60,0 \%)$, con procedencia del departamento de Santander (60,0\%), en un rango de edad de 3 a 5 años $(80,0 \%)$ y una condición corporal de 4 sobre 5 (80,0\%). Respecto a la temperatura de la canal no se encontraron temperaturas mayores de $40^{\circ} \mathrm{C}$. El grado de lesión más frecuente en los hígados de búfalos decomisados fue de tipo moderado (40,0\%) (tabla 2).

A. Bovinos

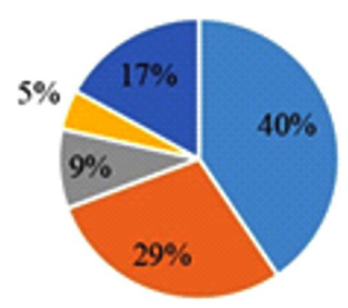

- Abscesos

= Distomatosis hepática

- Higado Graso

" Cirrosis

- Telangiectasia
En cuanto al decomiso por distomatosis hepática en bovinos, el mayor porcentaje de los casos se encontraron en el departamento de Santander (96\%, 42/44), dentro del cual los municipios con mayor proporción de decomisos por esta patología fue el municipio de Tona $(48 \%, 20 / 42)$, seguido de Guaca $(17 \%, 7 / 42)$, Piedecuesta (14\%, 6/41), San Andrés (9\%, 4/42), Girón (5\%, 2/42), Málaga $(5 \%, 2 / 42)$ y Santa Bárbara (2\%, 1/42), respectivamente (figura 4 ), con diferencias significativas entre los municipios $\left(\chi^{2}=50.03, P<0.05\right)$.

\section{Evaluación económica}

Luego de realizar el análisis económico, se determinó que la víscera con el mayor costo promedio en el mercado fue el hígado (16,71 USD), seguido del corazón (3,63 USD), riñón (3,50 USD), bazo (3,13 USD) y pulmón (3,05 USD), respectivamente. El costo promedio del total de vísceras rojas de un animal fue 47,30 USD.

Las mayores pérdidas económicas mensuales en el FRF estuvieron asociadas a decomisos de pulmón 4886 USD, seguido por decomisos de hígado 2639 USD, víscera roja completa 154,8 USD, riñón 11,2 USD, bazo 10,8 USD, y corazón 5,2 USD, respectivamente. El total de pérdidas económicas mensuales por decomiso de vísceras rojas fue de 7707 USD, para un total de pérdidas anuales estimadas de 92488 USD (figura 5). Finalmente, el valor promedio descontado por cada animal con decomiso independiente del órgano decomisado fue de 5,3 USD.

\section{Discusión}

Durante el periodo de estudio se sacrificaron un total de 8897 animales, en los cuales se realizó el decomiso de vísceras rojas en el 16,1\%. Este valor es inferior al histórico de decomisos informados en el FRF, en los que se mencionan promedios mensuales del $20 \%$ (Ramírez, 2019). Una disminución en el porcentaje

B. Búfalos

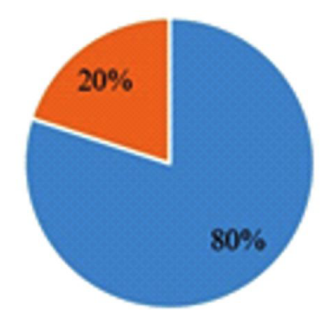
= Cirrosis

Figura 3. Causas de decomisos en hígados bovinos (A) y bufalinos (B) durante el estudio realizado en el FRF. Fuente propia. 
Tabla 2. Variables epidemiológicas analizadas y categorías encontradas con mayor frecuencia en los hígados decomisados durante el periodo de estudio. Fuente propia.

\begin{tabular}{|c|c|c|c|c|}
\hline \multirow{3}{*}{ Variable } & \multicolumn{4}{|c|}{ Categorías encontradas con mayor frecuencia } \\
\hline & \multicolumn{2}{|c|}{ Bovino } & \multicolumn{2}{|c|}{ Búfalo } \\
\hline & Categoría & $\%$ & Categoría & $\%$ \\
\hline Tiempo transporte & 6 a 10 horas & 52,9 & 1 a 5 horas & 60 \\
\hline Tiempo corrales & Más de 46 horas & 51 & 16 a 30 horas & 60 \\
\hline Raza & Bos indicus & 64,1 & - & - \\
\hline Sexo & Macho & 53,6 & Hembra & 60 \\
\hline Procedencia & Santander & 76,5 & Santander & 60 \\
\hline Edad & 3 a 5 años & 76,5 & 3 a 5 años & 80 \\
\hline Temperatura & Mayor de $40^{\circ} \mathrm{C}$ & 44 & Mayor de $40^{\circ} \mathrm{C}$ & 0 \\
\hline Condición corporal & $3 / 5$ & 80,4 & $4 / 5$ & 80 \\
\hline Patología & Abscesos & 40,5 & Hígado graso & 80 \\
\hline Grado lesión & Moderado & 79,1 & Moderado & 40 \\
\hline
\end{tabular}

de decomisos de vísceras rojas en el FRF puede estar asociado a la reciente implementación de un nuevo sistema de faenado por medio de un cajón de noqueo hidráulico, en el que se disminuye los movimientos bruscos de los animales durante este proceso (FAO, 2001). No obstante, variaciones temporales en los decomisos de vísceras rojas como se observó en recientes estudios de la provincia de Huancayo Perú (Arias et al., 2020), también pueden explicar estas variaciones.

Los resultados del estudio muestran que tanto en bovinos y búfalos los principales órganos decomisados fueron el pulmón e hígado, con porcentajes del 87,4\% - $88,3 \%$ y $11,1 \%-8,3 \%$, respectivamente. Estos resul-

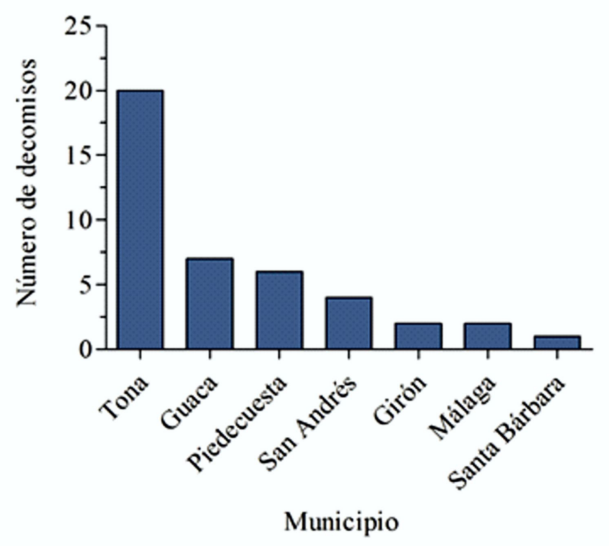

Figura 4. Municipios de Santander con mayor número decomiso de hígado por distomatosis hepática durante el estudio realizado en el FRF. Fuente propia. tados son similares a los observados por Ceccarelli et al., 2018, en la región central de Italia, en donde los pulmones y el hígado, fueron los órganos con mayor número de decomisos, con porcentajes del $64,8 \%$ y $31,2 \%$, respectivamente; pero difieren de los observados por Cedeño et al., 2012 y Burin et al., 2013 en la región de Nariño (Colombia) y Santa María (Brasil), en los cuales el órgano más decomisado fue el hígado $(67,9 \%$ y $40,4 \%)$, debido a la alta incidencia de distomatosis hepática. Estos resultados indican que la proporción de órganos decomisados en las plantas de beneficio varían dependiendo de las condiciones medioambientales y epidemiológicas de la zona, al igual que de las condiciones generales de infraestructura y funcionamiento de las plantas de beneficio (Oviedo et al., 2016).

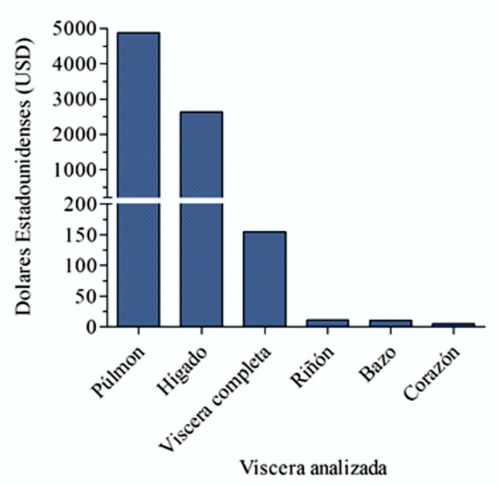

Figura 5. Estimado de pérdidas mensual por decomisos de vísceras rojas durante el estudio realizado en el FRF. Fuente propia. 
Los altos niveles de broncoaspiración y enfisema detectados en los pulmones decomisados de bovinos y bufalinos, son similares, a los reportados en el estado de Paraná (Brasil) (Negri y Okano, 2015), en donde ambas patologías fueron la principal causa de decomiso de este órgano, pero difieren de los reportados en el municipio de Montería (Colombia) (Oviedo et al., 2016), en donde la principal causa de decomiso fue el enfisema y las hemorragias. Según Oviedo et al., 2016, los altos niveles de broncoaspiración y enfisema podrían corresponder a falencias en el método de insensibilización, lo cual además de además de provocar grandes esfuerzos respiratorios en el animal durante su agonía, ocasiona la aspiración de contenido ruminal en el sistema respiratorio (Vivas y Buncic, 2004). Del mismo modo, la mayor proporción de decomisos en búfalos $\left(\chi^{2}, P<0.05\right)$ asociados principalmente a procesos de broncoaspiración, sugieren que los protocolos de prefaenado y faenado, deben ser ajustados a las características fisiológicas y anatómicas de esta especie a fin de disminuir el número de decomisos en este grupo de animales (Mendes y De Lima, 2011). De otro lado, estudios realizados en México demostraron que tiempos de espera en corrales mayor de 12 horas, favorecen la broncoaspiración de contenido ruminal, debido a la emesis post-aturdimiento (Ríos-Rincón et al., 2012). En este sentido los tiempos de espera en corrales de más de 46 horas registrados en el $79 \%$ y $86,8 \%$ de los bovinos y búfalos con decomisos de pulmón (tabla 1), pueden representar una de las principales causas broncoaspiración en los animales sacrificados, por lo que futuras intervenciones en el método de insensibilización y tiempos de espera en corrales deberían realizarse en el FRF con el fin de disminuir el número de decomisos de este órgano.

El hígado fue la segunda víscera más decomisada en ambas especies, para el caso de los bovinos, las principales causas de decomiso fueron los abscesos, seguido por distomatosis hepática y telangiectasia, mientras en los búfalos fue el hígado graso y la cirrosis. Estas diferencias pueden estar relacionadas con la comprobada resistencia de los búfalos a infecciones microbianas, distomatosis hepática y otras parasitosis (Negri y Okano, 2015; He et al., 2018). Con respecto a los resultados observados en bovinos, estos son diferentes a los observados en Nariño (Colombia) y Espiritu Santo (Brasil) (Dedeño, 2012; Tirandentes et al., 2017), donde la principal causa de decomiso fue la distomatosis hepática, indicando que la presentación de esta enfermedad varía significativamente dependiendo del área geográfica. En el presente estudio se observaron diferencias significativas en la proporción de hígados decomisados por distomatosis hepática en los dife- rentes municipios del departamento de Santander $\left(\chi^{2}\right.$, $P<0.05)$, con el $65,0 \%$ de los casos provenientes de los municipios de Tona y Guaca, ubicados a alturas superiores a los $1800 \mathrm{msnm}$, en donde las condiciones ambientales favorecen el desarrollo de caracoles del género Lymnaea (Carmona y Tort, 2016), principales huéspedes intermediarios de Fasciola hepatica. Estos resultados, indican que futuras estrategias de intervención en estas áreas geográficas deben ser realizadas con el fin de interrumpir el ciclo de vida de este parásito, y sus consecuentes impactos en la salud pública. De otro lado, los decomisos por abscesos hepáticos, puede estar asociado a la alta susceptibilidad de los bovinos al desarrollo de esta patología, producto de infecciones en órganos como los pre-estómagos, pulmón y pericardio, al igual que infecciones umbilicales, podo falángicas, metritis sépticas, entre otras (Altamirano, 2015). No obstante, cuadros de acidosis ruminal debido a malos procesos de alimentación, los cuales favorecen la infección hepática por Fusobacterium necrophorum y Actinomyces pyogenes (Nagaraja y Chengappa, 1998), también pueden explicar estos resultados.

Actualmente, el costo de los animales que se sacrifican en el FRF oscila alrededor de 46 millones de USD al año (Ramírez, 2019). En el presente estudio, las pérdidas económicas anuales asociadas al decomiso de vísceras rojas fueron de 92488 USD, lo cual representa el $0.2 \%$ del costo de los animales sacrificados en esta institución. De otro lado, las pérdidas económicas por cada animal con decomiso de vísceras rojas fueron de 5,3 USD; este valor, aunque parece poco significativo, representa alrededor del 5\% de la ganancia de los comercializadores intermediarios, los cuales adquieren una ganancia entre el 10 y el $20 \%$ sobre el precio del ganado en pie (Contexto Ganadero, 2015). El órgano que representó la mayor pérdida económica fue el pulmón, y entre las causas asociadas se refieren fallas en el proceso de faenado, largos tiempos de espera en corrales o enfermedades subdiagnosticadas (Oviedo, 2016). De otro lado, el segundo órgano que más pérdidas económicas generó fue el hígado con un estimado de 31683 USD anuales, diferente de lo reportado en otros mercados de Colombia y Costa Rica (Rojas y Cartín, 2016; Cedeño et al., 2012), donde valores de 67216 USD y 67438 USD, han sido reportados, respectivamente. Dicha variación puede deberse a diferencias en los procesos de beneficio y manejo animal implementado en cada frigorífico, al igual que a la alta incidencia de $F$. hepatica en los lugares de estudio (Arias-Pacheco et al., 2020).

Finalmente, el presente estudio concluyó que el pulmón es el principal órgano decomisado en bovinos y 
búfalos faenados en el FRF, al igual que la víscera con un mayor impacto económico en la ganancia de los comercializadores. Del mismo modo, se determinó que la mayoría de los casos de distomatosis hepática provienen de los municipios de Tona y Guaca, Santander; por tal motivo, los resultados aquí obtenidos deben ser tomados en cuenta para futuras intervenciones y trabajos de investigación con relación a reducir dichas problemáticas, y disminuir su impacto económico en la producción.

\section{Agradecimientos}

El presente trabajo fue realizado gracias a la cooperación científica del clúster ganadero establecido entre el Frigorífico Rio Frio y la Universidad Cooperativa de Colombia (UCC), sede Bucaramanga. Finalmente, agradecemos a todos los administrativos y operarios del FRF quienes colaboraron en la realización del trabajo.

\section{Referencias}

Altamirano M. Incidencia de las principales alteraciones hepáticas macroscópicas en bovinos faenados en el camal municipal de Ambato. Universidad Técnica de Ambato. 2015. Disponible en: http://repo.uta.edu.ec/bitstream/123456789/8684/1/ Tesis $\% 2025 \% 20$ Medicina\%20Veterinaria\%20y\%20Zootecnia\%20-CD\%20311.pdf.

Arias-Pacheco C, Lucas, JR, Rodríguez A, Córdoba D, Lux-Hoppe EG. Economic impact of the liver condemnation of cattle infected with Fasciola hepatica in the Peruvian Andes. Trop Anim Health Prod, 2020. https://doi.org/10.1007/s11250-020-02211-y

Burin AP, de Avila EF, Karsten JK, Scortegagna A, Dörr AC, Laerte JN. Perdas econômicas oriundas das condenações de vísceras bovinas em matadouros de Santa Maria, Rio Grande do Sul. $R$ Bras Ci Vet, 2013;(20):99-103. http://dx.doi.org/10.4322/ $\underline{\text { rbcv. } 2014.053}$

Carmona C, Tort JF. Fasciolosis in South America: epidemiology and control challenges. J Helminthol, 2016;91(2):1-11. https://doi. org/10.1017/S0022149X16000560

Ceccarelli M, Leprini E, Sechi P, Francesca MI, Luca G, Enzo G, Beniamino T. Analysis of the causes of the seizure and destruction of carcasses and organs in a slaughterhouse in central Italy in the 2010-2016 period. Ital. J Food Saf, 2018;7(1): 6899. https:// doi.org/10.4081/ijfs.2018.6899

Cedeño D, Martínez G, Cilima R. Principales causas de decomiso de vísceras rojas en bovinos en el frigorífico del municipio de Pasto. REVIP, 2012;(2):8-15. https://revistas.udenar.edu.co/index. $\mathrm{php} / \mathrm{revip} / \mathrm{article} / \mathrm{view} / 380$

Contexto Ganadero. Top 5 de los países que más producen carne de res. Contexto Ganadero. 2015. Disponible en: https://www. contextoganadero.com/internacional/top-5-de-los-paises-quemas-producen-carne-de-res.
Dólar-Colombia. Precio del dólar en noviembre 2018. Disponible en: https://www.dolar-colombia.com/mes/2018-11

FAO. (2001). Directrices para el Manejo, Transporte y Sacrificio Humanitario del Ganado. Food and Agriculture Organization of the United Nations Regional Office for Asia and the Pacifi. Disponible en: http://www.fao.org/3/x6909s/x6909s00. $\underline{\text { htm\#Contents }}$

Frigorífico Río Frío (FRF). Reseña Frigorífico Río Frío. (2020). Disponible en: http://www.frigorificoriofrio.com/secciones-38-s/ resena-historica-htm.

He C, Mao Y, Zhang X, Li H, Lu K, Fu Z, Liu J. High resistance of water buffalo against reinfection with Schistosoma japonicum. Vet Parasitol, 2018;261:18-21. https://doi.org/10.1016/i.vetpar.2018.08.00

Instituto Colombiano Agropecuario (ICA). Censo Pecuario Nacional 2019. Instituto Nacional Agropecuario. 2017. Disponible en: https://www.ica.gov.co/areas/pecuaria/servicios/epidemiologia-veterinaria/censos-2016/censo-2018

Instituto Nacional de vigilancia y control de medicamentos y alimentos (INVIMA). Plantas de beneficio, desposte, desprese y acondicionadores que cumplen con los requisitos sanitarios exigidos en el decreto 1500 de 2007, decreto 2270 de 2012 y resoluciones reglamentarias. 2018. Disponible en: https:// www.invima.gov.co/images/pdf/informate/Plantas_Beneficio/ PLANTAS-DE-BENEFICIO-DESPOSTE-Y-DESPRESE-AUTORIZADA-POR-EL-INVIMA-PARA-SU-FUNCIONAMIENTO-A-ENERO15-DE-2019-AS-ASP.pdf

Mendes J, De Lima-Francisco C. Evaluación de canales y calidad de la carne de búfalo. Tecnología en Marcha, 2011;24(5):36-59. file://C:/Users/Usuario/Downloads/Dialnet-EvaluacionDeCanalesYCalidadDeLaCarneDeBufalo-4835760.pdf

Ministerio de salud y protección social (Minsalud). Resolución 240 de 2013. Ministerio de Salud y Protección Social. 2013. Disponible en: https://docs.supersalud.gov.co/PortalWeb/Juridica/ OtraNormativa/R_MSPS_0240_2013.pdf.

Nagaraja TG, Chengappa MM. Liver abscesses in feedlot cattle: a review. J Anim Sci, 1998;76(1):287-98. https://doi. org/10.2527/1998.761287x

Oviedo TS, Vargas-Viloria M, Cardona JA. Frecuencia de lesiones macroscópicas en pulmones de bovinos tipo exportación faenados en el frigorífico del municipio de Montería, Colombia. Rev Med Vet Zoot, 2016;(63):30-38. https://doi.org/10.15446/ rfmvz.v63n1.56902

Ramírez AF. Caracterización de decomisos de vísceras rojas orientadas al proceso de exportación en el Frigorífico Río Frío, del municipio de Floridablanca-Santander. [Bucaramanga]: Tesis de pregrado, Universidad Cooperativa de Colombia; 2019. Disponible en: https://repository.ucc.edu.co/ handle/20.500.12494/8149

Ríos-Rincón FG, Estrada-Angulo A, Hernández-Bautista J, Pérez-Linares C, Portillo-Loera JJ, Robles-Estrada JC. Factores que influyen en la emesis postaturdimiento en bovinos. Rev Mex Cienc Pecu, 2012;3:343-356. http://www.scielo.org.mx/pdf/rmcp/v3n3/ v3n3a6.pdf 
Rojas D, Cartín JA. Prevalence of Fasciola hepatica and financial losses due to liver discards at three class A abattoirs in Costa Rica. Agron. Costarricense. 2016; 40(2):53-62. https://www.scielo. sa.cr/pdf/ac/v40n2/0377-9424-ac-40-02-00053.pdf

Tirandentes LA, Falçoni FM, Vieira NP. Principais causas de condenações de fígados de bovinos em matadouros frigoríficos do sul do estado do Espirito Santo no período de 2011 a 2016. Revista Dimensão Acadêmica, 2017;(2):37-52. https://multivix. edu.br/wp-content/uploads/2018/09/revista-dimensao-academica-v02-n02-artigo03.pdf
Vivas AL, Buncic S. Potential for use of hide-carcass microbial counts relationship as an indicator of process hygiene performance of cattle abattoirs. Food Prot Trends, 2004;24(11):814-820.

Xiaojuan L, Xibi F, Ping J, Hang X, Haibin Y, Mengjiao Z, Yunzhi P, et al. AGPAT6 gene EX1_303T > C and EX12_299G > A mutations and associations with economic traits of Chinese Simmentalcross cattle. Arch Anim Breed, 2016;59: 301-307. https://doi. org/10.5194/aab-59-301-2016

Felipe Ramírez: https://orcid.org/0000-0001-5943-934X Arturo Cárdenas: https://orcid.org/0000-0002-2167-9038 Víctor Arcila: https://orcid.org/0000-0002-5889-601X Ruby Cristancho: https://orcid.org/0000-0003-4026-2227 Jeiczon Jaimes: https://orcid.org/0000-0001-5839-8523 